$=0$ at $22{ }^{\circ} \mathrm{C}$ and $\Delta \mathrm{S}=0$ at $113^{\circ} \mathrm{C}$ ) and residual contributions, $\Delta \mathrm{H}_{\text {res }}$ and $\Delta \mathrm{S}_{\text {res }}$, then the latter turn out to be independent of the temperature. They presumably represent some contribution from the protein molecule itself apart from the hydrophobic interaction. Furthermore, both $\Delta \mathrm{H}_{\text {res }}$ and $\Delta \mathrm{S}_{\text {res }}$ are large and positive, as would be expected if the unfolded protein is anything like a random coil in which the favourable packing interactions that exist in the native state have been disrupted. Even more significantly, the per residue value of $\Delta S_{\text {res }}$ turns out to be about the same for several simple globular proteins. Privalov had already shown that the plots of temperature against $\Delta S$ for unfolding per residue for several typical small proteins tend to intersect near 110 ${ }^{\circ} \mathrm{C}$, which is a consequence of Baldwin's considerations.

Baldwin does not comment on the molecular basis of his observations; what he has to say is independent of that. This matter has been discussed at length by many authors and is controversial. I still believe that the Frank and Evans 'iceberg' model of 40 years ago is essentially correct: when it is transferred into water the non-polar hydrocarbon molecule induces, in the layer of water immediately surrounding it, a cage of more or less fully hydrogen-bonded water molecules (the iceberg). The large decrease in entropy that is known to accompany the transfer is accounted for by the increase in order accompanying the formation of the iceberg. As the temperature is raised the iceberg melts bit by bit - whence the large contribution to the specific heat. Indeed, this notion has been dealt with quantitatively by S.J. Gill et al. (J. phys. Chem. 89, $3758-3761 ; 1985)$. This interpretation is also supported by molecular simulations of methane and neon in water, which invariably show a layer of structured water surrounding the non-polar solute molecule. Some of the attempts to explain these observations in other ways seem to me to be ludicrous, to say the least.

Baldwin's success with his model is remarkable in several respects. He wishes to explain the interaction with water of a non-polar group, $\mathrm{R}$, in a molecule $\mathrm{R}-\mathrm{X}$, where $X$ can be a single atom, such as a halogen (as in methyl chloride) or a group of atoms (as in ethanol or acetic acid, where $\mathrm{X}$ is $-\mathrm{OH}$ or $-\mathrm{CO}-\mathrm{OH}$ ). To do this he studies the behaviour of the molecule $\mathrm{R}-\mathrm{H}$ in water, as if the nature of the group $\mathrm{X}$ makes no difference to the interaction between water and $\mathrm{R}$. That this will work at all is perhaps not remarkable if $\mathrm{X}$ contains only a few atoms, but in Baldwin's

THE picture of vortices on the cover of the 22-28 January issue of Nature was produced by Jim Salem (Thinking Machines Corporation, Cambridge, Massachusetts) and Stephen Wolfram (University of Illinois).

\title{
Sale by Wheatstone Laboratory, London
}

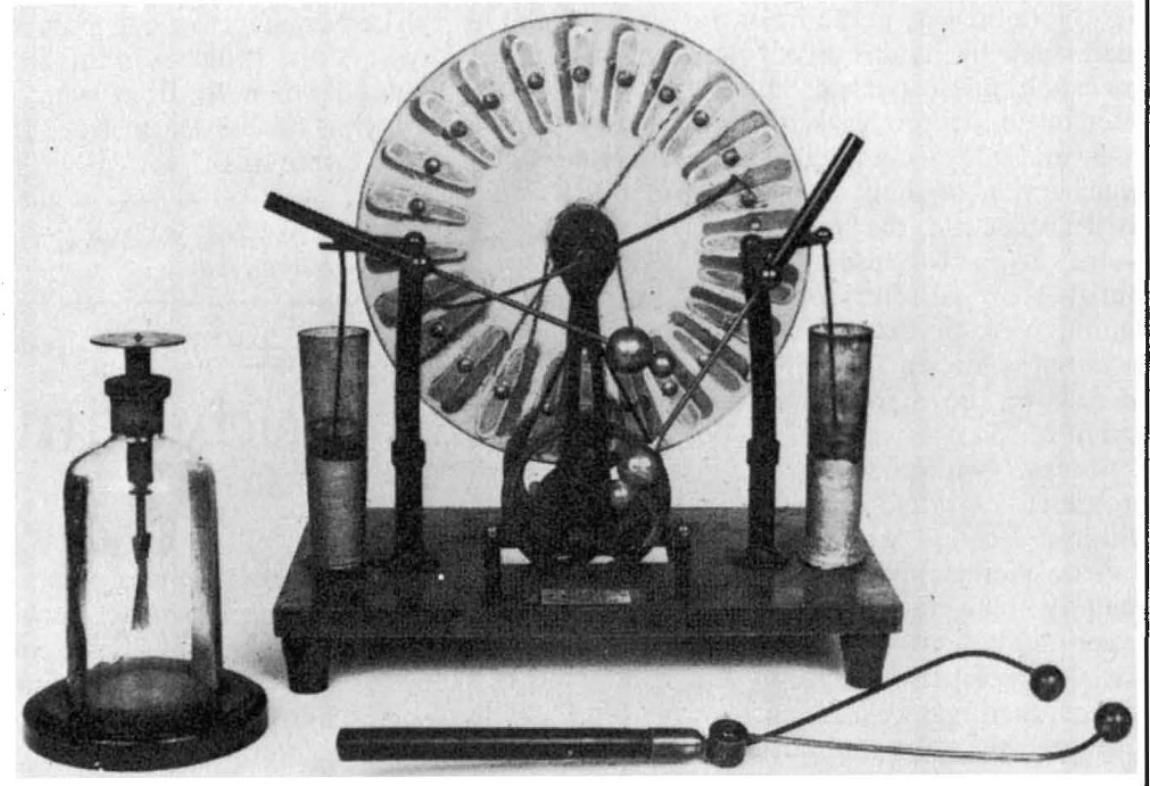

A NineteENTH century electrostatic generator (Wimshurst pattern) by Becker \& Co., with a gold-leaf electroscope and a discharging fork. The Wimshurst machine can generate thousands of volts with a few turns of the handle, producing sparks between the electrodes. These items are included in a sale of scientific and philosophical apparatus by the Wheatstone Laboratory, King's College, London, at Christie's, South Kensington, London, on 5 March at 10.30 a.m.

case $\mathrm{X}$ is the polypeptide backbone, with enormous steric and other influences that could easily interfere with the success of the approach. Evidently, they do not.

The point has also been made that the interior of a folded protein molecule is more likely to resemble a solid than a liquid, so that it is wrong to use the liquid hydrocarbon as the analogue with the clusters of non-polar residues that are known to exist in protein interiors. Baldwin's success shows that this cannot be an important consideration in the phenomenon that concerns him here.

But there is a fly in the ointment. The liquid hydrocarbon model fails almost completely when one attempts to extend it to the effects of pressure on protein folding. Just as temperature effects on the unfolding equilibrium are determined by the enthalpy change, $\Delta \mathrm{H}$, of the process, so the pressure effects are determined by the volume change, $\Delta V$, that accompanies the unfolding. Studies on several proteins clearly show that $\Delta V$ for unfolding is positive at low pressures but becomes negative at pressures above 1-2 kbar. (This shows that the compressibilities of the folded and unfolded forms must be very different, just as their heat capacities are different.) On the other hand, studies of the effect of pressure on the solubility in water of non-polar substances just as clearly show the opposite behaviour: $\Delta V$ for the transfer from non-polar to aqueous environment is negative at low pressures but becomes positive at pressures above
$1-2$ kbar. This failure of the hydrophobic liquid model is mentioned in passing by Baldwin, and workers in the field are probably aware of it in a general way, but the fact is that this discrepancy could well be devastating to the whole approach. Volume and enthalpy changes are equally fundamental properties of the unfolding process, and no model can be considered acceptable unless it accounts for the entire thermodynamic behaviour.

It is, of course, much easier to study the effects of temperature on systems than to study pressure effects. Thermostatic baths are relatively cheap and very easy to work with, whereas high-pressure apparatus (I refer to pressures of up to $5 \mathrm{kbar}$, which is really not very high) is expensive and awkward to use. It is easy, therefore, to sweep this discrepancy under the rug. A great deal more work has been done on temperature effects, and there are many more data to work with. But one is reminded of the story of the drunk who, one dark night, has lost his keys. He is seen looking for them under a street light. When asked where he lost them, he points across the street, where all is dark. "Why, then, are you looking for them here?" He replies, "Because there is more light here". Until more searching is done in the darkness of high-pressure studies, our understanding of the hydrophobic effect must be considered quite incomplete.

Walter Kauzmann is Emeritus Professor of Chemistry at Princeton University, Princeton, New Jersey 08544, USA. 